\title{
Rate-Distortion Behavior at Low Distortion for Densely Sampled Gaussian Data
}

\author{
David L. Neuhoff \\ EECS Department \\ University of Michigan \\ Ann Arbor, MI 48109 \\ neuhoff@eecs.umich.edu
}

\author{
S. Sandeep Pradhan \\ EECS Department \\ University of Michigan \\ Ann Arbor, MI 48109 \\ pradhanv@eecs.umich
}

\begin{abstract}
It is well known that for discrete-time, stationary sources, most lossy source coding techniques have operational rate-distortion functions that approach the Shannon ratedistortion function with respect to squared error to within an additive constant as distortion approaches zero. With the goal of investigating similar phenomena for continuous-time sources, this paper investigates the low-distortion performance of distributed coding of continuous-time, stationary, Gaussian sources based on high-rate sampling. It is found that for bandlimited sources and nonbandlimited sources whose spectra have sufficiently light, e.g., exponentially decreasing, tails, distributed source coding is asymptotically as good as centralized coding in the small distortion regime. On the other hand, for spectra with tails that decay as a power (greater than one) of frequency, it is found that for small distortions the distributed rate-distortion function is a constant times larger than the Shannon rate-distortion, where the constant decreases as the power increases. For example, it is approximately 1.2 when the power is 2 . The conclusion is that for a stationary Gaussian source and asymptotically small distortion, the ratio of the distributed to centralized rate-distortion function is a function of the weight of the tail of the source spectrum. In the process of finding the ratio, the low distortion form of the centralized rate-distortion function is found for sources whose spectra have exponential and power law tails.
\end{abstract}

\section{INTRODUCTION}

High-resolution theory, c.f. [1], shows that for almost any stationary discrete-time source with finite variance and for large rate $r$, the Shannon distortion-rate function with respect to squared-error distortion has the form

$$
D(r) \cong c 2^{-2 r}
$$

where $c$ is some positive constant that depends on the source. Equivalently, the Shannon rate-distortion function has the form

$$
R(d) \cong-\frac{1}{2} \log _{2} d+\frac{1}{2} \log _{2} c .
$$

(Actually, the theory shows that the ratio of the left- and righthand sides of (1) asymptotically approaches one as $r \rightarrow \infty$, and the difference between the left- and right-hand sides of (2) asymptotically approaches zero as $d \rightarrow 0$.) Moreover, for most classes of lossy source codes ${ }^{1}$, including fixedand variable-rate scalar and vector quantization, as well as transform coding, the operational distortion-rate function for

\footnotetext{
${ }^{1}$ The only exception known to the authors is fixed-rate uniform scalar quantization [2].
}

the given source and class satisfies (1) in the same asymptotic sense except for some other constant $c$ that depends on the source and the class of codes.

On the other hand, for continuous-time sources, much less is known about distortion-rate and rate-distortion functions with respect to squared-error distortion. For a bandlimited, stationary, Gaussian source with a spectrum that is flat over a band of positive frequencies of width $b$, in his classic paper [3], Shannon found the rate-distortion function to be

$$
\mathcal{R}(d)=-\frac{b}{2 \pi} \log d+\frac{b}{2 \pi} \log _{2} P,
$$

where $P$ is the power of the source, which has the same form as (2).

About the only other known rate-distortion function is the following inverse water pouring parametric characterization for a stationary Gaussian source, due to Kolmogorov [4] (see also [5, p. 117]):

$$
\begin{gathered}
\overline{\mathcal{R}}(\theta) \triangleq \frac{1}{2 \pi} \int_{-\infty}^{\infty} \max \left\{\frac{1}{2} \log _{2} \frac{S(\Omega)}{\theta}, 0\right\} d \Omega \\
\overline{\mathcal{D}}(\theta) \triangleq \frac{1}{2 \pi} \int_{-\infty}^{\infty} \min \{S(\Omega), \theta\} d \Omega,
\end{gathered}
$$

where $S(\Omega)$ is the power spectral density of the source and $\theta$

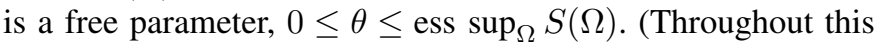
paper, caligraphic font will indicate continuous-time rate and distortion, overbars will indicate parametric rate and distortion formulas, and we focus exclusively on stationary, Gaussian sources and squared-error distortion.)

Berger [5, p. 138] has used the above result to show that for a bandlimited stationary Gaussian source with nonflat spectrum and for small values of $d$, the rate-distortion function has the same form as in (2) and (3):

$$
\mathcal{R}(d) \cong-a \log _{2} d+b
$$

where $a$ and $b$ are known constants.

On the other hand, it is has been shown in [6], see also [5, p. 145], that for a nonbandlimited, stationary Gauss-Markov source with spectrum

$$
S(\Omega)=\frac{2}{\Omega^{2}+1},
$$


and for small $d$, the rate-distortion function has the form

$$
\mathcal{R}(d) \cong \frac{1}{2 \ln 2}\left(\frac{8}{\pi^{2} d}-1\right),
$$

which has a very different form than (2) and (3). Thus we see that unlike discrete-time, the continuous-time rate-distortion function can have very different forms in the low-distortion region.

Recent work of the authors [7]-[10] has parametrically characterized the operational rate-distortion function for a continuous-time, stationary, Gaussian source, squared-error distortion, and two types of lossy coding based on high-rate sampling and discrete-time coding of the resulting samples.

First, for ideal lossy distributed coding of the samples, using the Berger-Tung bound [11], it was found that the following rate-distortion performance is attainable [7], [9], [10]:

$$
\begin{gathered}
\overline{\mathcal{R}}_{B T}(\theta) \triangleq \frac{1}{4 \pi} \int_{-\infty}^{\infty} \log \left(\frac{S(\Omega)}{\theta}+1\right) d \Omega \\
\overline{\mathcal{D}}_{B T}(\theta) \triangleq \frac{1}{2 \pi} \int_{-\infty}^{\infty} \frac{S(\Omega) \theta}{S(\Omega)+\theta} d \Omega
\end{gathered}
$$

for any $\theta \in[0, \infty)$. Let $\mathcal{R}_{B T}(d)$ denote the corresponding operational rate-distortion function, which is an upper bound to the best possible performance of distributed coding based on high-rate sampling that we call the BT rate-distortion function. Note that to obtain this result, it was necessary to impose technical conditions on the tails of the source spectrum and autocorrelation function in order that the sampled process have autocorrelation function and power spectral density in $\mathbb{L}_{2}$, and also to hypothesize an additional coding dimension. In particular, in [7], [9], [10] the main coding dimension is space and the additional dimension is time. That is, for each sampled location in space, a separate, i.e., distributed, encoder operates on a temporal block of samples taken at this location. A decoder then operates on the encodings of a spatial block of samples to decode all samples in the corresponding spatiotemporal block. Since in the present paper there is no attention to actual code operation, it is not necessary to consider an additional dimension and the principal dimension is considered to be time.

Second, for orthogonal transform coding operating on highrate samples, the operational rate-distortion function is found to be characterized as follows [8]-[10]:

$$
\begin{aligned}
& \overline{\mathcal{R}}_{\mathcal{T}, \mathcal{Q}}(\gamma) \triangleq \frac{1}{2 \pi} \int_{-\infty}^{\infty} \tilde{R}_{\mathcal{Q}}(\psi(\gamma S(\Omega))) d \Omega \\
& \overline{\mathcal{D}}_{\mathcal{T}, \mathcal{Q}}(\gamma) \triangleq \frac{1}{2 \pi} \int_{-\infty}^{\infty} S(\Omega) \psi(\gamma S(\Omega)) d \Omega
\end{aligned}
$$

for any $\gamma \leq 0$, where $\mathcal{Q}$ denotes a specific family of scalar quantizers, e.g., uniform scalar quantizers with entropy coding, $\tilde{R}_{\mathcal{Q}}(d)$ denotes the lower convex hull of the operational ratedistortion function, and where it is assumed that $\tilde{R}_{\mathcal{Q}}(d)$ satisfies some technical conditions, referred to as goodness, and either $\tilde{R}_{\mathcal{Q}}(d)$ is strictly convex or the power spectral density has no flat spots in the sense that for every $s>0$, the
$\{\Omega: S(\Omega)=s\}$ is a set of measure zero. A somewhat more involved parametric characterization applies when neither of the last two assumptions holds, but the process is bandlimited. Let $\mathcal{R}_{\mathcal{T}, \mathcal{Q}}(d)$ denote the operational rate-distortion function for orthogonal transform coding based on high-rate sampling and the family of quantizers $\mathcal{Q}$.

Numerical evaluations using (6)-(9) for the Gauss-Markov source with spectrum (4) suggest that both $\mathcal{R}_{B T}(d) / \mathcal{R}(d)$ and $\mathcal{R}_{\mathcal{T}, \mathcal{Q}}(d) / \mathcal{R}(d)$ approach constants larger than one as $d$ goes to zero. This behavior is rather different than in discretetime, where, as mentioned earlier, we almost always see the difference between an operational rate-distortion function and the Shannon rate-distortion approaching a constant, and consequently their ratio approaches one. The goal of the present paper is to analytically derive the low distortion form of the operational distortion-rate function in the case of distributed coding and to compare this to the low rate form of the Shannon rate-distortion function. Results are provided for several source spectra. In the process, we also find the low distortion form of the centralized rate-distortion function for some spectra that have not previously been analyzed. The case of transform coding is left to future work.

\section{LOW Distortion PERFormancE OF DISTRIBUTED CODING}

\section{A. Bandlimited sources}

Consider first a stationary Gaussian source whose spectrum is bandlimited in the sense that $S(\Omega)>0$ when and only when $\Omega \in B$, where $B$ is a closed and bounded set. Moreover, assume that $S(\Omega)$ is a continuous function on $B$, so that it has a nonzero minimum value. Then from (6),

$$
\begin{aligned}
\overline{\mathcal{R}}_{B T}(\theta)= & \frac{1}{4 \pi} \int_{B} \log \left(\frac{S(\Omega)}{\theta}+1\right) d \Omega \\
= & \frac{1}{4 \pi} \int_{B} \log \frac{S(\Omega)}{\theta} d \Omega \\
& +\frac{1}{4 \pi} \int_{B} \log \left(1+\frac{\theta}{S(\Omega)}\right) d \Omega \\
= & \overline{\mathcal{R}}(\theta)+O(\theta),
\end{aligned}
$$

where $O(\theta)$ denotes a quantity that remains within a bounded factor of $\theta$ as $\theta \rightarrow 0$. It follows that

$$
\overline{\mathcal{R}}_{B T}(\theta)-\overline{\mathcal{R}}(\theta) \rightarrow 0 \text { as } \theta \rightarrow 0 .
$$

Similarly, from (7),

$$
\begin{aligned}
\overline{\mathcal{D}}_{B T}(\theta) & =\frac{1}{2 \pi} \int_{B} \frac{\theta S(\Omega)}{S(\Omega)+\theta} d \Omega \\
& =\frac{1}{2 \pi} \int_{B} \theta d \Omega-\frac{1}{2 \pi} \int_{B} \frac{\theta^{2}}{S(\Omega)+\theta} d \Omega \\
& =\overline{\mathcal{D}}(\theta)-o(\theta),
\end{aligned}
$$

where $o(\theta)$ denotes a quantity whose ratio to $\theta$ goes to zero as $\theta$ goes to zero. It follows that

$$
\frac{\overline{\mathcal{D}}_{B T}(\theta)-\overline{\mathcal{D}}(\theta)}{\theta} \rightarrow 0 \text { as } \theta \rightarrow 0 \text {. }
$$


Using (10) and (11), one can then show that

$$
\mathcal{R}_{B T}(d)-\mathcal{R}(d) \rightarrow 0 \text { as } d \rightarrow 0 .
$$

That is, at asymptotically low distortions there is no loss in distributed coding relative to centralized coding. Considering distortion-rate functions instead of rate-distortion functions, one can also show

$$
\frac{\mathcal{D}_{B T}(r)}{\mathcal{D}(r)} \rightarrow 1 \text { as } r \rightarrow \infty
$$

The essentially optimal behavior of distributed coding at low distortions for bandlimited sources can be explained as follows. The forward test channel that achieves the BergerTung distributed coding operational rate-distortion function has the following structure:

$$
\hat{X}(\Omega)=c(\Omega)(X(\Omega)+Q(\Omega)),
$$

where $X(\Omega)$ is the fourier transform of the source sample function $x(t), Q(\Omega)$ is the Fourier transform of the forward quantization noise, and $c(\Omega)$ is a minimum mean squared error estimation coefficient. Due to the constraint of distributed compression, the quantization noise at different sources are independent, making $Q(\Omega)$ constant (white). In contrast, in the centralized coding case, $Q(\cdot)$ can be chosen to be anything. In other words, the only performance loss of distributed coding as compared to centralized coding is due to its inability to color the forward quantization noise in the ideal way. In particular, in the centralized case the spectrum of the quantization noise should be chosen so that the spectrum of the minimum mean squared error (MMSE) is as flat as possible (i.e., inverse-water pouring). When distortion is small, the water level is indeed exactly flat. The value of the spectrum of MMSE at frequency $\Omega$ is given by

$$
\frac{S(\Omega) S_{Q}(\Omega)}{S(\Omega)+S_{Q}(\Omega)} .
$$

In the distributed case, $S_{Q}(\cdot)$ is constrained to be flat, whereas in the centralized case, $S_{Q}(\cdot)$ is chosen to make $\frac{S(\Omega) S_{Q}(\Omega)}{S(\Omega)+S_{Q}(\Omega)}$ flat. When the distortion is close to 0 , the difference between the two spectra is small, as

$$
\frac{S(\Omega) S_{Q}(\Omega)}{S(\Omega)+S_{Q}(\Omega)} \approx S_{Q}(\Omega) .
$$

Therefore, distributed coding loses little or nothing in comparison to centralized coding when distortion is small.

\section{B. Exponential spectrum}

Consider next a stationary Gaussian source with the exponential spectrum

$$
S(\Omega)=e^{-|\Omega|},
$$

which although not bandlimited has a very light tail. For this source, the parametric form of the rate-distortion function can be computed to $\mathrm{be}^{2}$

$$
\begin{aligned}
& \overline{\mathcal{R}}(\theta)=\frac{(\ln \theta)^{2}}{4 \pi \ln 2}, \\
& \overline{\mathcal{D}}(\theta)=-\frac{\theta}{\pi} \ln \theta+\frac{\theta}{\pi},
\end{aligned}
$$

which can be used to find the distortion-rate function

$$
\mathcal{D}(r)=\frac{1}{\pi} e^{-\sqrt{4 \pi R \ln 2}}(1+\sqrt{4 \pi R \ln 2}) .
$$

The rate-distortion function $\mathcal{R}(d)$, i.e., the inverse of the above, can be expressed in terms of a secondary branch of the Lambert $\mathrm{W}$ function. Although the latter does not have a closed form expression, there is a useful expansion [12] with which it can be shown that

$$
\begin{aligned}
\mathcal{R}(d) & =\frac{1}{4 \pi \ln 2}\left(\ln \frac{e}{d \pi}+\ln \ln \frac{e}{d \pi}-1+o(1)\right)^{2} \\
& =\frac{1}{4 \pi \ln 2}\left(\ln ^{2} \frac{e}{d \pi}\right)(1+o(1)),
\end{aligned}
$$

where $o(1)$ denotes a quantity that goes to zero as $d$ goes to zero.

One can also compute the parametric form of the BT ratedistortion function:

$$
\begin{aligned}
\overline{\mathcal{R}}_{B T}(\theta) & =\frac{(\ln \theta)^{2}}{4 \pi \ln 2}+\frac{\pi}{12 \ln 2}-\frac{\theta}{2 \pi \ln 2}+o(\theta) \\
& =\overline{\mathcal{R}}(\theta)+\frac{\pi}{12 \ln 2}-\frac{\theta}{2 \pi \ln 2}+o(\theta), \\
\overline{\mathcal{D}}_{B T}(\theta) & =\frac{\theta}{\pi} \ln \theta+\frac{\theta}{\pi} \ln (1+\theta) \\
& =\overline{\mathcal{D}}(\theta)-\frac{\theta}{\pi}(1-\ln (1+\theta)) \\
& =\overline{\mathcal{D}}(\theta)-\frac{\theta}{\pi}+o(\theta) .
\end{aligned}
$$

One can then use the above to relate the BT and Shannon rate-distortion functions. Specifically, one can show

$$
\limsup _{d \rightarrow 0}\left(\mathcal{R}_{B T}(d)-\mathcal{R}(d)\right) \leq \frac{\pi}{12 \ln 2}=0.378,
$$

and

$$
\lim _{r \rightarrow \infty} \frac{\mathcal{D}_{B T}(r)}{\mathcal{D}(r)}=1 .
$$

Notice that although (15) is not sufficient to imply that the lim sup in (14) is zero, it might well be so, because the excess rate evidenced in (12) is mitigated to some extent by the decreased distortion in (13). In any case, we see that for a Gaussian source with an exponential density, at low distortions distributed coding loses little if anything to centralized coding.

\section{Gauss-Markov source}

For a Gauss-Markov source with spectrum given in (4) and rate-distortion function given in (5) the BT distributed ratedistortion function was found in [7], [9], [10] to be

$$
\mathcal{R}_{B T}(d)=\frac{1}{2 \ln 2}\left(\frac{1}{d}-1\right),
$$

\footnotetext{
${ }^{2}$ The derivations here and for subsequent results are omitted.
} 
which differs from the low distortion approximation to $\mathcal{R}(d)$ in (5) only in that the factor one multiplying $1 / d$ is larger than the factor $8 / \pi^{2}=0.81$. In other words, distributed coding requires approximately $19 \%$ more rate than centralized coding. This is a significantly larger difference than for a bandlimited or exponential spectra.

\section{Spectrum with power law tail}

Suppose now that the spectrum $S(\Omega)$ of a stationary Gaussian source is monotonically decreasing for all sufficiently large $\Omega$ and has a power law tail in the sense that

$$
S(\Omega) \Omega^{u} \triangleq C(\Omega) \rightarrow C \text { as } \Omega \rightarrow \infty
$$

for some $u>1$ and $C>0$. Equivalently,

$$
S(\Omega)=C(\Omega) \Omega^{-u}=C \Omega^{-u}(1+o(1)) .
$$

where $o(1) \rightarrow 0$ as $\Omega \rightarrow \infty$.

The parametric form of the rate-distortion function can be shown to be

$$
\begin{gathered}
\overline{\mathcal{R}}(\theta)=\frac{u C^{1 / u}}{2 \pi \ln 2} \frac{1}{\theta^{1 / u}}+o\left(\frac{1}{\theta^{1 / u}}\right) \\
\overline{\mathcal{D}}(\theta)=\frac{C^{1 / u}}{\pi} \frac{u}{u-1} \theta^{\frac{u-1}{u}}(1+o(1)) .
\end{gathered}
$$

Using these, the rate-distortion function can be shown to be

$$
\begin{gathered}
\mathcal{R}(d)=\frac{1}{2 \ln 2} \frac{1}{\pi^{\frac{u}{u-1}}} u^{\frac{u}{u-1}}(u-1)^{-\frac{1}{u-1}} C^{\frac{1}{u-1}} \frac{1}{d^{\frac{1}{u-1}}} \\
+o\left(\frac{1}{d^{\frac{1}{u-1}}}\right),
\end{gathered}
$$

which gives rate to within a small percentage, but not to within a small additive constant.

For $u=2$ and $C=2$, and the above reduce to

$$
\begin{gathered}
\overline{\mathcal{R}}(\theta)=\frac{\sqrt{2}}{\pi \ln 2} \frac{1}{\sqrt{\theta}}+o\left(\frac{1}{\sqrt{\theta}}\right) \\
\overline{\mathcal{D}}(\theta)=\frac{2 \sqrt{2}}{\pi} \sqrt{\theta}+o(\sqrt{\theta})
\end{gathered}
$$

and

$$
\mathcal{R}(d)=\frac{4}{\pi^{2} \ln 2} \frac{1}{d}+o\left(\frac{1}{d}\right),
$$

which match the corresponding formulas for the GaussMarkov source.

To learn the small distortion behavior of the BT ratedisortion function for distributed coding, we first consider the asymptotic ratio of $\overline{\mathcal{R}}_{B T}(\theta)$ to $\overline{\mathcal{R}}(\theta)$ in the limit of small $\theta$ by considering the ratio of $\overline{\mathcal{R}}_{B T}(\theta)$ to $\theta^{-\frac{1}{u}}$ and show

$$
\frac{\overline{\mathcal{R}}_{B T}(\theta)}{\theta^{-\frac{1}{u}}} \rightarrow \frac{1}{2 \pi \ln 2} C^{\frac{1}{u}} \int_{0}^{\infty} \ln \left(\frac{1}{x^{u}}+1\right) d x
$$

Therefore,

$\overline{\mathcal{R}}_{B T}(\theta)=\theta^{-\frac{1}{u}} \frac{1}{2 \pi \ln 2} C^{\frac{1}{u}} \int_{0}^{\infty} \ln \left(\frac{1}{x^{u}}+1\right) d x(1+o(1))$.
As in the case of rate, we consider the asymptotic ratio of $\overline{\mathcal{D}}_{B T}(\theta)$ to $\overline{\mathcal{D}}(\theta)$ in the limit of small $\theta$ by considering the ratio of $\overline{\mathcal{D}}_{B T}(\theta)$ to $\theta^{1-\frac{1}{u}}$. The result is

$$
\frac{\overline{\mathcal{D}}_{B T}(\theta)}{\theta^{1-\frac{1}{u}}} \rightarrow \frac{1}{\pi} C^{\frac{1}{u}} \int_{0}^{\infty} \frac{1}{1+x^{u}} d \Omega .
$$

Therefore,

$$
\overline{\mathcal{D}}_{B T}(\theta)=\theta^{\frac{u-1}{u}} \frac{1}{\pi} C^{\frac{1}{u}} \int_{0}^{\infty} \frac{1}{1+x^{u}} d x(1+o(1)) .
$$

From the parametric expressions, one may show

$$
\mathcal{R}_{B T}(d)=\frac{1}{2 \pi \ln 2}\left(\frac{C A(u)}{\pi}\right)^{\frac{1}{u-1}} B(u) \frac{1}{d^{\frac{1}{u-1}}}+o\left(\frac{1}{d^{\frac{1}{u-1}}}\right),
$$

where

$$
A(u)=\int_{0}^{\infty} \frac{1}{1+x^{u}} d x \text { and } B(u)=\int_{0}^{\infty} \ln \left(\frac{1}{x^{u}}+1\right) d x .
$$

For $u=2$ and $C=2$, these become

$$
\begin{gathered}
\overline{\mathcal{R}}_{B T}(\theta)=\frac{1}{\sqrt{2 \theta} \ln 2}+o\left(\frac{1}{\sqrt{\theta}}\right) \\
\overline{\mathcal{D}}_{B T}(\theta)=\frac{1}{\sqrt{2}} \sqrt{\theta}+o(\sqrt{\theta})
\end{gathered}
$$

and

$$
\widetilde{\mathcal{R}}_{B T}(d)=\frac{1}{2 \ln 2} \frac{1}{d}+o\left(\frac{1}{d}\right),
$$

which match the corresponding results for the Gauss-Markov source.

For small $d$, we see that

$$
\frac{\mathcal{R}_{B T}(d)}{\mathcal{R}(d)} \rightarrow B(u)\left(\frac{(u-1) A(u)}{u^{u}}\right)^{\frac{1}{u-1}} \text { as } d \rightarrow 0 .
$$

For $u=2$, the limiting ratio equals $\frac{\pi^{2}}{8}$, which again matches the result for Gauss-Markov. Moreover, it can be shown that as $u$ decreases to one, the limiting expression on the right side of the above goes to infinity. Thus, as $u \rightarrow 1$, the asymptotic low-distortion ratio of the BT rate-distortion function to the Shannon rate-distortion function becomes arbitrarily large.

\section{Discussion AND CONCLUSIONS}

It was already understood that the low distortion behavior of the rate-distortion function relates to the tail of the spectrum, with a light-tailed spectrum, such as bandlimited or exponential, having rate-distortion function that approaches infinity more slowly than for a heavy-tailed spectrum such as that of the Gauss-Markov source. Thus, the latter is easier to encode. The results of this paper indicate that, in addition, the lighter the tail, the less distributed coding loses to centralized coding at low distortion. In particular, for a bandlimited spectrum with no tail, there is no loss, and for an exponentially decaying tail, which is very light, there is asymptotically no loss. On the other hand, for a power law tail, the asymptotic low-distortion ratio of $\mathcal{R}_{B T}(d)$ to $\mathcal{R}(d)$ approaches a constant greater than one, which grows without bound as the power approaches 1 . Although the $\mathcal{R}_{B T}(d)$ is known only to be an upper bound to 
the operational rate-distortion function for distributed coding of high-rate samples, it is our belief that it gives a good indication of the latter. Interestingly, the results of this paper indicate that only the tail of the spectrum affects the limiting ratio. That is, it is not affected by the low frequency part of the spectrum. Finally, we mention that the low distortion form of the centralized rate-distortion function was found for sources whose spectra is exponential or has a power law tail. To our knowledge, these have not previously appeared in the literature.

Some interesting questions remain. For example, it would be nice to know the precise dividing line between spectra such that the limiting ratio of $\mathcal{R}_{B T}(d)$ to the Shannon ratedistortion function is one and spectra whose limiting ratio is greater than one. Also, it would be nice to know if there are spectra whose tails are so heavy that the limiting ratio grows without bound as distortion decreases.

\section{ACKNOWLEDGMENT}

This work was supported by NSF grants CCF-0915619, CCF-1116021, and CCF-0830438.

\section{REFERENCES}

[1] R.M. Gray and D.L. Neuhoff, "Quantization," IEEE Trans. Inform. Thy, vol. IT-44, pp. 2325-2383, Oct. 1998.

[2] D. Hui and D.L. Neuhoff, "Asymptotic analysis of ptimal fixed-Rate uniform scalar quantization," IEEE Trans. Inform. Thy., vol. IT-47, pp.957977, Mar. 2001.

[3] C.E. Shannon, "A mathematical theory of communication," Bell Syst. Tech. J., vol. 27, pp. 379-423, 623-656, 1948.

[4] N. Kolmogorov, "On the Shannon theory of information transmission in the case of continuous signals," IEEE Trans. Inform. Thy., vol. 2, pp. 102 $108,1956$.

[5] T. Berger, Rate distortion theory: A mathematical basis for data compression. Englewood Cliffs: Prentice Hall, 1971.

[6] T.J.Goblick, "Theoretical limitations on the transmission of data from analog source," IEEE Trans. Inform. Thy., vol. IT-11, pp. 558-567, Oct. 1965.

[7] D.L. Neuhoff and S.S. Pradhan, "An upper bound to the rate of ideal distributed source coding of densely sampled data," Proc. IEEE Int. Conf. on Acoustics, Speech, and Signal Proc. (ICASSP), 2006.

[8] S.S. Pradhan and D.L. Neuhoff, "Transform coding of densely sampled Gaussian data," Proc. IEEE Int. Symp. Inform. Thy., Nice, France, pp. 1111-1114, June 2007.

[9] D.L. Neuhoff and S.S. Pradhan, "Information rates of densely sampled Gaussian data," Proc. IEEE Intl Symp. Inform. Thy., pp. 2877-2881, St. Petersberg, Russia, Aug. 2011.

[10] D.L. Neuhoff and S.S. Pradhan, "Information rates of densely sampled Gaussian data: distributed vector quantization and scalar quantization with transforms," submitted to IEEE Trans. Inform. Thy., Dec. 2011.

[11] T. Berger, "Multiterminal Source Coding," Information Theory Approach to Communication, (CISM Courses and Lecture Notes No. 229), G. Longo, Ed., Wien and New York: Springer-Verlag, 1977.

[12] R.M.Corless, G.H. Gonnet, D.E.G. Hare, D.J. Jeffrey, and D.E. Knuth, "On the Lambert W function," Advances in Computational Mathematics vol. 5, no. 1, pp. 329-359, 1996. 